independantly assessed the level of evidence (EL) using SIGN (Scottish Intercollegiate Guidelines Network) methodology (http.//www. sign.ac.uk). CRBSI rates were expressed as per 1000CVC days.

Results The search strategy yielded 3142967 hits. Combination searches using 'IF' and 'Child' reduced this to 2993. 14 studies were read in detail; 5 were excluded due to containing purely adult data or where data on PIF could not be analysed seperately. Nine studies were included in our review, 5 of CRBSI prevention and 4 of CRSBI treatment. 8 studies used ethanol alone and 1 reported taurolidine use (Table). 4 studies reported success in prevention of recurrent line sepsis. 2 thrombotic episodes were reported.

Conclusion The data for the use of ethanol line locks are limited of poor methodoligical quality and of lower EL (there are no RCT's or well designed cohort studies). However ethanol locks appear to be an effective therapy in CRBSI prevention and treatment. With only 1 study of taurolidine locks no comparison can be made with ethanol locks. Future well designed studies are warranted to compare these two treatments.

Disclosure of Interest None Declared.

\section{PWE-188 PATIENT SELECTION FOR PEG INSERTION: ARE WE MAKING THE RIGHT DECISIONS?}

doi:10.1136/gutjnl-2013-304907.476

1."R Hammond, ${ }^{2} \mathrm{~J}$ Cotton, ${ }^{2} \mathrm{~J}$ Fyall, ${ }^{2} \mathrm{~K}$ Turnbull, ${ }^{2} \mathrm{~J}$ Tait. ${ }^{1}$ Medical School, University of Dundee; ${ }^{2}$ Dept. of Gastroenterology, Ninewells Hospital and Medical School, Dundee, UK

Introduction Careful patient selection is key in the success of percutaneous endoscopic gastrostomy (PEG) procedures ${ }^{1}$. The 30 day mortality rate post insertion is an indicator of appropriate selection in those who are chosen for PEG, but reveals nothing about the patients judged unsuitable for PEG. We evaluated our decision making behind patient selection based on outcomes in those with a PEG inserted and those without.

Methods The study identified all patients referred for specialist nurse-led PEG assessment between Jan 2007 - Dec 2011 within our centre. Data regarding age, sex, diagnosis, indication for PEG, date of referral, reason for non-insertion and RIG referrals were stored prospectively on a clinical database and analysed retrospectively. Patients were stratified into groups and mortality in each examined. Further information regarding cause of death and alternative feeding methods were obtained for selected patients from paper and electronic patient notes.

Results A total of 555 PEG referrals were received with $38 \%$ of all referrals to the PEG team resulting in PEG non-insertion. The 30 day mortality rate following PEG insertion was on average $6.1 \%$; this reduced from $8.6 \%$ in 2007 to $2.2 \%$ in $2011.50 \%$ of all patients in the non-insertion group had a CVA as their diagnosis. $47 \%$ of all non-insertion patients and $83 \%$ of insertion patients were alive 120 days after referral. Reasons for non-insertion were grouped into unfit ( $\mathrm{n}=98,46 \%$ of total), improved $(\mathrm{n}=44,21 \%)$, contraindicated $(\mathrm{n}=34,16 \%)$ and refused ( $\mathrm{n}=26,12 \%) .74 \%$ of those deemed unfit died within 30 days of referral, and $93 \%$ of those judged to be improving were alive at 4 months post-referral. RIG referrals were arranged in 19 of 34 patients contraindicated against a PEG procedure. Patient or family refusal was the main reason for non-insertion in $12 \%$ of the non-insertion group. 12 notes were examined in patients who died in 60-180 days following PEG referral: 9 had evidence of NG feeding and 3 received RIGs. Extensive MDT input was evident. 4 patients were re-referred to the service for a second assessment if the best option was unclear.

Conclusion Patient selection for PEG will continue to be complex. The nurse-led PEG assessment team, in conjunction with other MDT members, make well-informed and justifiable decisions, based on the low 30 day mortality rate post insertion, and that reasons against insertion correlate with how patient condition progresses. Alternative feeding methods are employed in the non-insertion group to combat ongoing nutritional needs. Lack of information on quality of life is the main limitation to the conclusion.

Disclosure of Interest None Declared.

\section{REFERENCE}

1. Kurien M, McAlindon ME, Westaby D, Sanders DS. Percutaneous endoscopic gastrostomy (PEG) feeding. Bmj. 2010 May 7; 340(may07 2):c2414-c2414.

\section{PWE-189 SGLT3A AND GLP-1 DISPLAY A DIURNAL RHYMICITY OF MRNA EXPRESSION IN MOUSE PROXIMAL SMALL BOWEL}

doi:10.1136/gutjnl-2013-304907.477

1. ${ }^{*} \mathrm{R}$ Hewett, ${ }^{1 P}$ O'Brien, ' $\mathrm{C}$ Corpe. 'Diabetes and Nutritional Sciences Division, King's College, London, UK

Introduction A diurnal cycle is one that recurs every 24 hours Many physiological processes such as blood sugar levels exhibit diurnal variation. Such processes are under the control of central and peripheral clock genes that have an endogenous rhythmicity, but are entrained (synchronised) by external light and food input cues. Diurnal rhythmicity of gene expression has previously been described in intestinal nutrient/energy transporters such as Sodium Glucose co- transporter-1 (SGLT-1) and Glucose transporter 5 (GLUT-5). SGLT-1 mediates the glucose induced release of glucosedependent insulinotropic peptide (GIP) and Glucagon like peptide 1 (GLP-1) and therefore has an additional sugar sensing role. Mouse SGLT-3a does not transport sugar and is thus postulated to be purely a sugar sensor. SGLT- 3a, GLP-1 or GIP have not previously been demonstrated to have a diurnal rhythmicity of expression.

Methods Sixteen C57BL/6J mice were fed ad libitum under conditions of 12-hour light/dark cycles. Half the animals were randomly euthanized in the morning and half were euthanized in the evening. Duodenal and jejunal tissues were isolated from the carcasses and messenger RNA (mRNA) extracted. Complementary DNA (cDNA) was synthesised from mRNA and underwent real-time (quantitative) PCR. Expression levels for each gene were expressed as a ratio to two housekeeping genes (HMBS and HPRT-1) Relative quantification of gene expression was done using the comparative CT $(2-\Delta \Delta \mathrm{CT})$ method.

Results In keeping with previous studies the sugar transporters GLUT-5 and SGLT-1 ( $p<0.005)$ and the clock genes Cry- 2 and Bmal-1 $(p<0.01)$ displayed a diurnal rhythmicity of expression in both tissues. For the first time SGLT-3a was shown to display a marked (more than double) up-regulation of mRNA expression in the evening compared to the morning in both duodenum and jejunum ( $p<0.005)$. GLP-1 exhibited approximately twice the levels of expression in the evening than in the morning but this was not statistically significant. GIP failed to show any diurnal rhythmicity of expression.

Conclusion Demonstrated for the first time was a diurnal rhythmicity of SGLT-3a and GLP-1 expression. It is postulated that sugar sensing by SGLT-3a has an important role in mediating beneficial downstream sequelae such as gut peptide hormone release. Dysregulation of such mechanisms may play important roles in metabolic diseases such as diabetes.

Disclosure of Interest None Declared.

\section{PWE-190 OUTCOME OF INVESTIGATIONS FOR IRON DEFICIENCY ANAEMIA IN MEN UNDER 50 YEARS}

doi:10.1136/gutjnl-2013-304907.478

'O D Patani, 'S Bharathi, 1."S Khalid. 'Gastroenterology, Warrington and Halton Hospitals NHS Trust, Warrington, UK

Introduction Iron-deficiency anaemia (IDA) occurs in $2-5 \%$ of men and postmenopausal women in the developed world. IDA is 